SHORT REPORT

\title{
Intraventricular cytarabine in a case of idiopathic hypertrophic pachymeningitis
}

\author{
I Kleiter, V H Hans, G Schuierer, J Marienhagen, P Hau, H Schütz, U Bogdahn, A Steinbrecher
}

J Neurol Neurosurg Psychiatry 2004;75:1346-1348. doi: 10.1136/jnnp.2003.024653

Idiopathic hypertrophic chronic pachymeningitis (IHCP) is characterised by inflammatory fibrotic thickening of the dura mater. Long term management is controversial. A 28 year old man with craniospinal IHCP and prominent lymphocytic meningitis is reported. Cerebrospinal fluid and histological examination suggested a CD4+ T cell driven process and $B$ cell stimulation. After surgical, tuberculostatic, and immunosuppressive treatment failed to control the progressive meningeal hypertrophy, causing severe headache and neurological disability, the disease process eventually abated with intraventricular cytarabine treatment.

l: diopathic hypertrophic chronic pachymeningitis (IHCP) is a rare disorder of unknown origin. The main clinical features consist of cranial nerve palsies, headache, and ataxia. Histological examination typically reveals granulomatous inflammatory infiltrates and fibrotic thickening of the dura mater. Autoimmune, neoplastic, and infectious causes have been suggested. ${ }^{1}$ IHCP is subdivided into cranial, spinal, and craniospinal types, the latter being the least common. ${ }^{2}$ Various therapeutic strategies have been recommended, including surgical removal of hypertrophied tissue and the use of immunosuppressive agents. ${ }^{1-5}$ However, the optimal regimen for long term management remains elusive. ${ }^{2}{ }^{4}$ We describe the clinical course of a patient suffering from craniospinal IHCP whose condition ultimately improved with intraventricular cytarabine.

\section{CASE REPORT}

In 1991, a slight bilateral hearing loss was diagnosed in a 19 year old man. At least 10 years earlier, recurrent periorbital furuncles of the left upper and lower lid and the nose had been removed surgically without further problems. His medical and social history was otherwise unremarkable.

In May 1996, he complained of acute visual loss in the right eye, which recovered incompletely following pulse steroid therapy. Four months later, bilateral deterioration of vision occurred (visual acuity: OD 0.03, OS 0.8), and an audiogram showed bilateral hypacusis of -40 to $-50 \mathrm{~dB}$. Physical examination, including neurological assessment, was otherwise normal. A meningeal mass close to the tuberculum sellae and extending onto the clivus was demonstrated on magnetic resonance imaging (MRI). Cerebrospinal fluid (CSF) analysis revealed pleocytosis of $748 / \mathrm{mm}^{3}$ (97\% mononuclear cells) and protein of $242 \mathrm{mg} / \mathrm{dl}$ (normal <45). The $\mathrm{CSF} /$ serum ratio for albumin was $39.1 \times 10^{-3}$ (normal $<5.6 \times 10^{-3}$ ), for immunoglobulin (Ig)A $42.0 \times 10^{-3}$, and for IgG $23.9 \times 10^{-3}$. According to Reiber, ${ }^{6}$ Ig$\mathrm{CSF} /$ serum ratios in this patient not exceeding $27.2 \times 10^{-3}$ for IgA and $23.9 \times 10^{-3}$ for IgG would be explained by the extent of blood-brain barrier disruption alone. Thus the findings were consistent with intrathecal immunoglobulin production restricted to IgA. Neither oligoclonal bands nor malignant cells were detected. CSF microscopy, cultures, and polymerase chain reaction for Mycobacterium tuberculosis were negative. Serum and CSF glucose and angiotensin converting enzyme were normal. ECG, chest $x$ ray, and abdominal ultrasound were unremarkable. Routine blood analysis, including erythrocyte sedimentation rate, was normal.

Therapeutic interventions with cefotaxime $(8 \times 6 \mathrm{~g}$ intravenously) and subsequent tuberculostatic quadruple chemotherapy were unsuccessful. The patient underwent partial surgical removal of the retrosellar lesion. Microscopic examination showed meningothelial proliferation and a marked inflammatory reaction (fig $1 \mathrm{~A}$ and $\mathrm{IC}$ ). Immunohistochemistry for Ki-67 revealed a proliferation index of approximately 5\% (fig 1D). Mitoses were inconspicuous. The infiltrating mononuclear cells were in the main CD45 positive. Immunohistochemical reactions showed both CD45R0 expressing $\mathrm{T}$ lymphocytes and areas containing plasma cells (fig 1B). B lymphocytes (CD20) were sparse. There was no evidence of abscess or granuloma formation, giant cells, or vasculitis. Neither neutrophilic nor eosinophilic infiltration could be observed. These histopathological findings were consistent with the diagnosis of a lymphoplasmacyte-rich meningioma (WHO grade I). However, chronic meningitis with reactive meningothelial proliferation could not be excluded on morphological grounds.

While clinical symptoms remained stable, annual follow up MRI showed progressive thickening of the dura with rostrocaudal spreading (fig 2A). Computed tomography (CT) documented a chronic pansinusitis, and biopsy of the nasal mucosa revealed non-specific chronic inflammation. Thoracic $\mathrm{CT}$ and bone marrow biopsy showed no abnormalities.

In February 2000, further deterioration of left eye vision was unresponsive to pulse steroid treatment. Despite intensive immunosuppressive therapy in the following months-consisting of oral steroids, intravenous bolus cyclophosphamide $(6 \times 1 \mathrm{~g})$, and subsequent intrathecal methotrexate $(6 \times 15 \mathrm{mg})$-visual acuity deteriorated further, while CSF pleocytosis and raised protein persisted.

In December 2000, the patient complained of holocephalic headache, nausea, and occasional vomiting when standing up. The right eye was amaurotic. On the left side, only perception of light was preserved, with concomitant bilateral optic disc atrophy. Bilateral sensorineural hearing deficit measured -60 to $-70 \mathrm{~dB}$. The neurological status was otherwise normal. Lumbar puncture yielded an opening pressure of $480 \mathrm{~mm} \mathrm{H} \mathrm{H}_{2} \mathrm{O}, 245$ cells $/ \mathrm{mm}^{3}$ (89\% lymphocytes), protein $749 \mathrm{mg} / \mathrm{dl}$, lactate $45.5 \mathrm{mg} / \mathrm{dl}$, and a CSF/plasma glucose ratio of $13 \%$. Oligoclonal bands were present. T or B cell monoclonality was excluded. FACS analysis of lymphocyte subpopulations revealed normal amounts of $\mathrm{T}$ cell $(\mathrm{CD} 3+), \mathrm{CD} 4+$, and CD8+ subsets and NK cells $(\mathrm{CDl6}+\mathrm{CD} 56+)$ in the CSF and the peripheral blood. Relative numbers of $\mathrm{B}$ cells (CD19+CD20+) were $28 \%$ in CSF (normal range $<3 \%$ ) and $4 \%$ in blood. Monoclonal 

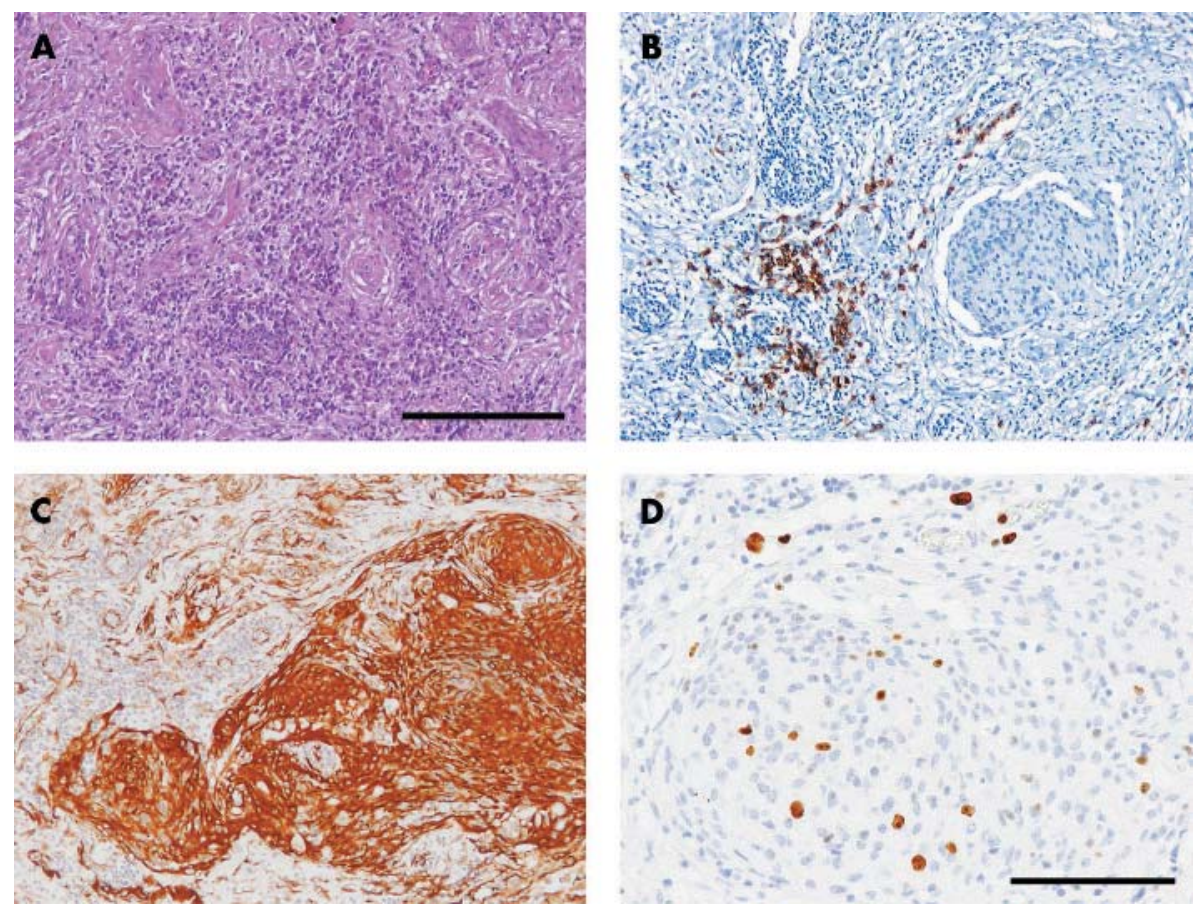

Figure 1 (A) A photomicrograph of the surgical specimen showing perivascular and diffuse lymphoplasmocellular infiltrates and vascularised fibrous tissue surrounding meningothelial proliferates (haematoxylin and eosin). (B) Some mononuclear cells can be identified as CD138 positive plasma cells. (C) Meningothelial cells expressing vimentin are arranged in sheets and whorls. (D) Several nuclei of meningothelial cells labelled using an antibody against Ki-67. Panels $A, B$, and C are at the same magnification: bar $=200 \mu \mathrm{m}$; panel D: bar $=100 \mu \mathrm{m}$.

gammopathy was excluded. A laboratory screen for systemic autoimmune disease and proinflammatory cytokines was uneventful. Serology and PCR analyses of CSF and serum for a broad spectrum of viruses, bacteria, fungi and protozoa were negative. Multiple cultures from serum, urine, and CSF were sterile for bacteria including all known subtypes of mycobacteria and fungi. Somatostatin receptor (SSTR) scintigraphy with $\left[{ }^{111} \mathrm{In}\right]$-octreotide revealed only a weak signal at the skull base and around the cervical cord.
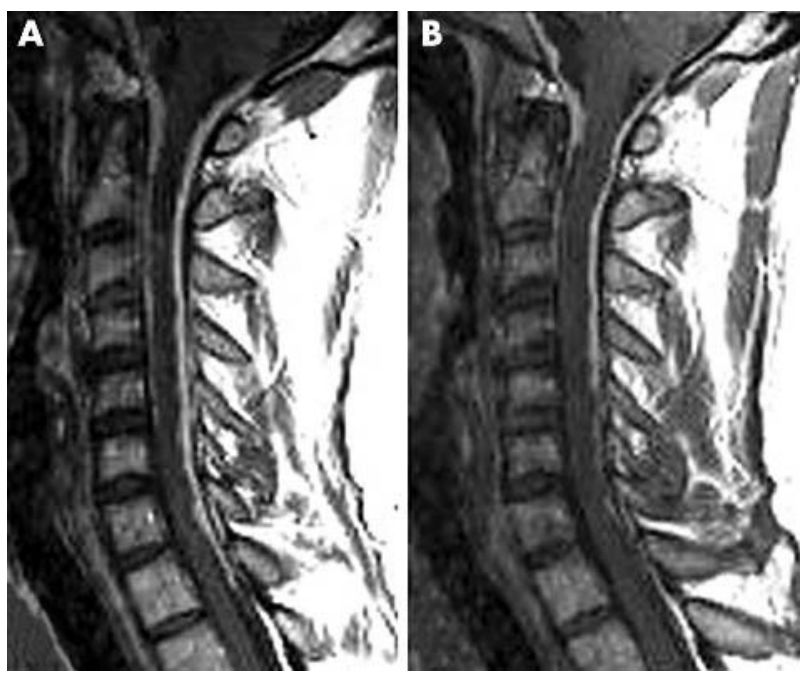

Figure 2 (A) Pretreatment sagittal T1 weighted magnetic resonance imaging after gadolinium enhancement: homogeneously enhancing meninges extend throughout the posterior fossa, completely entrapping the upper cervico-thoracic spinal cord. (B) Partial reduction of gadolinium enhancing masses at 27 months after initiation of cytarabine treatment.
At this point the presumptive diagnosis of IHCP with chronic lymphocytic meningitis was made. Experimental intrathecal cytotoxic treatment was initiated, consisting of cytarabine $(4 \times 80 \mathrm{mg})$ with triamcinolone-acetonide $(80 \mathrm{mg}$ at first and third applications). Within two weeks, CSF pathology improved dramatically. Cytarabine $(40 \mathrm{mg})$ was given weekly for six months using an Ommaya reservoir and then slowly tapered. CSF ( $15 \mathrm{ml}$ ) was removed twice weekly and acetazolamide ( $1 \mathrm{~g} / \mathrm{d}$ ) was given orally. Except for mild transient nausea, the treatment was well tolerated and headaches improved continuously. Subsequent MRI examinations showed stable disease with partial regression of meningeal hypertrophy (fig 2B). At a 27 month follow up the patient reported being well. Vision on the left eye had improved slightly and residual hearing capacity remained stable. Headache had ceased and all CSF indices were normal. Subsequently, intraventricular cytarabine was replaced by oral azathioprine. Long term treatment may require the additional implantation of a ventriculo-peritoneal shunt.

\section{DISCUSSION}

The clinical and neuroradiological features of this patient are consistent with the diagnosis of craniospinal IHCP associated with aseptic meningitis and raised intracranial pressure. As the syndrome of hypertrophic pachymeningitis may have various causes, the diagnosis of IHCP remains one of exclusion. It is tempting to hypothesise that the retrosellar lesion, originally described as a lymphoplasmacyte-rich meningioma, was the anatomical origin of the pachymeningitis. However, our case highlights the well described difficulty of discriminating definitively between a lymphoplasmacyte-rich meningioma growing en plaque and a diffuse inflammatory meningothelial hyperplasia-even after biopsy. ${ }^{78}$ In our patient, the histopathological findings of meningothelial proliferations surrounded by a chronic inflammation may be interpreted either as a strong 
lymphoplasmacytic reaction to a primary neoplasm (that is, a meningioma) or a meningeal response to a lymphocytic pachymeningitis of unknown origin. Exact differentiation between reactive meningothelial hyperplasia and neoplasia is probably impossible on clinical and histopathological grounds alone. The number of Ki67 positive nuclei in a solid meningothelial area is suspicious but by no means proof of a true neoplasm. The result of the SSTR scintigraphy rather favours the explanation of a secondary meningeal reaction as meningiomas usually express high levels of SSTR. ${ }^{9}$

The patient had suffered from facial furuncles and long lasting pansinusitis, possible risk factors for chronic pachymeningitis. ${ }^{4}$ However, their significance in our case is unclear. There was no proof of other known causative factors such as trauma, bacterial, viral, or fungal infections, neoplasia, or granulomatous diseases-that is, Wegener's granulomatosis, sarcoidosis, or tuberculosis.

While a progressive rostro-caudal meningeal thickening has been observed in some, albeit not all, cases of IHCP, ${ }^{23}{ }^{10} \mathrm{a}$ persistent CSF pleocytosis $\left(>100\right.$ cells $\left./ \mathrm{mm}^{3}\right)$ is a rather uncommon feature of IHCP. ${ }^{10}{ }^{11}$ The high proportion of B cells in the CSF, prominent plasma cell infiltration in the meninges, and intrathecal immunoglobulin production indicate oligoclonal or polyclonal B cell stimulation in the CNS, possibly initiated by a $\mathrm{T}$ cell driven immune process. Interestingly, lymphocytapheresis has been reported to improve IHCP. ${ }^{12}$

Nevertheless, the optimal long term treatment strategy for IHCP is unknown. ${ }^{2}{ }^{4}$ While high dose steroids have a transient effect in many cases, radiotherapy is probably not beneficial. ${ }^{1}$ Surgery can relieve mass effects, especially when spinal compression occurs. ${ }^{2}$ To the best of our knowledge, this is the first report describing the successful use of cytarabine in IHCP which was used because standard immunosuppressive treatment with corticosteroids, cyclophosphamide, and intrathecal methotrexate failed to improve the condition. The antimetabolite cytarabine is a standard cytotoxic agent for the intrathecal therapy of neoplastic meningitis, in particular of lymphoid origin. We hypothesise that cytarabine may be a promising drug in targeting both the inflammatory and proliferative component of the disease process, irrespective of the nature of the initiating injury. Importantly, adverse side effects were not seen in our patient.

\section{Conclusions}

The dramatic improvement in the chronic meningitis and the arrest of the meningoproliferative process with intraventricular cytarabine suggest that this approach might be considered in selected patients suffering from IHCP with marked pleocytosis, after exclusion of an infectious aetiology. Further reports on the successful use of this treatment are required before making a general recommendation. Both immunosuppressive strategies and treatment of raised intracranial pressure, however, need to be pursued early in the course of IHCP to avoid irreversible cranial nerve impairment.

\section{Authors' affiliations}

I Kleiter, P Hau, U Bogdahn, A Steinbrecher, Department of Neurology, University of Regensburg, Regensburg, Germany

G Schuierer, Institute of Neuroradiology, University of Regensburg

J Marienhagen, Department of Nuclear Medicine, University of

Regensburg

V H Hans, Institute of Neuropathology, University of Bonn, Bonn,

Germany

H Schütz, Department of Neurology, Städtische Kliniken Frankfurt am

Main - Höchst, Germany

Competing interests: none declared

I Kleiter and V H Hans contributed equally to this work.

Correspondence to: Dr I Kleiter, University of Regensburg, Department of Neurology, Universitätsstrasse 84, 93042 Regensburg, Germany; ingo.kleiter@klinik.uni-regensburg.de

Received 29 July 2003

Revised 3 December 2003

Accepted 7 December 2003

\section{REFERENCES}

1 Masson C, Henin D, Hauw JJ, et al. Cranial pachymeningitis of unknown origin: a study of seven cases. Neurology 1993;43:1329-34.

2 Dumont AS, Clark AW, Sevick RJ, et al. Idiopathic hypertrophic pachymeningitis: a report of two patients and review of the literature. Can J Neurol Sci 2000;27:333-40.

3 Voller B, Vass K, Wanschitz J, et al. Hypertrophic chronic pachymeningitis as a localized immune process in the craniocervical region. Neurology 2001;56:107-9.

4 Hatano N, Behari S, Nagatani T, et al. Idiopathic hypertrophic cranial pachymeningitis: clinicoradiological spectrum and therapeutic options. Neurosurgery 1999;45:1336-42; discussion 42-4.

5 Choi IS, Park SC, Jung YK, et al. Combined therapy of corticosteroid and azathioprine in hypertrophic cranial pachymeningitis. Eur Neurol 2000;44:193-8.

6 Reiber H. Flow rate of cerebrospinal fluid (CSF) - a concept common to normal blood-CSF barrier function and to dysfunction in neurological diseases. J Neurol Sci 1994;122:189-203.

7 Yamaki T, Ikeda T, Sakamoto Y, et al. Lymphoplasmacyte-rich meningioma with clinical resemblance to inflammatory pseudotumor. Report of two cases. J Neurosurg 1997;86:898-904.

8 Pittella JE, da Costa CC, Giannetti AV, et al. October 2000: a 47 year old man with long-standing progressive tetraparesis. Brain Pathol $2001 ; 11: 261-2$.

9 Bohuslavizki KH. Somatostatin receptor imaging: current status and future perspectives. J Nucl Med 2001;42:1057-8.

10 Berger JR, Snodgrass S, Glaser J, et al. Multifocal fibrosclerosis with hypertrophic intracranial pachymeningitis. Neurology 1989;39:1345-9.

11 Wouda EJ, Vanneste JA. Aspecific headache during 13 years as the only symptom of idiopathic hypertrophic pachymeningitis. J Neurol Neurosurg Psychiatry 1998;64:408-9.

12 Yamamoto T, Goto K, Suzuki A, et al. Long-term improvement of idiopathic hypertrophic cranial pachymeningitis by lymphocytapheresis. Ther Apher 2000;4:313-16. 